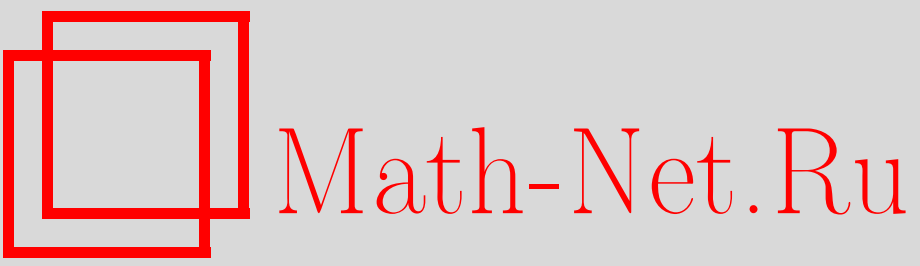

А. В. Болсинов, И. А. Тайманов, О примере интегрируемого геодезического потока с положительной топологической энтропией, УМH, 1999, том 54, выпуск 4, 157-158

DOI: https://doi.org/10.4213/rm184

Использование Общероссийского математического портала Math-Net.Ru подразумевает, что вы прочитали и согласны с пользовательским соглашением

http: //www . mathnet.ru/rus/agreement

Параметры загрузки:

IP: 18.208 .226 .222

26 апреля 2023 г., 13:12:25 


\title{
О ПРИМЕРЕ ИНТЕГРИРУЕМОГО ГЕОДЕЗИЧЕСКОГО ПОТОКА С ПОЛОЖИТЕЛЬНОЙ ТОПОЛОГИЧЕСКОЙ ЭНТРОПИЕЙ
}

\author{
А. В. БОЛСИНОВ, И. А. ТАЙМАНОВ
}

Геодезический поток на римановом многообразии $M^{n}$ с метрикой $g_{j k}$ - это гамиль тонова система на кокасательном расслоении $T^{*} M^{n}$ с функцией Гамильтона $H(x, p)=\frac{1}{2} g^{j k}(x) p_{j} p_{k}$ и скобками Пуассона $\left\{x^{j}, p_{k}\right\}=\delta_{k}^{j},\left\{x^{j}, x^{k}\right\}=\left\{p_{j}, p_{k}\right\}=0$, где $\left\{x^{j}\right\}$ - координаты на $M^{n}$, а $\left\{p_{k}\right\}$ - координаты на слоях кокасательного расслоения, связанные с касательными векторами $\dot{x}$ к $M^{n}$ преобразованием Лежандра: $p_{k}=g_{j k} \dot{x}^{j}$.

Поверхности уровня $L_{c}=\{H=c \neq 0\}$ попарно диффеоморфны, и ограничения геодезического потока на них гладко траекторно изоморфны: если $(\gamma(t), \dot{\gamma}(t))$ - траектория в $L_{c}$ и $\nu(t)=\gamma(\sqrt{k} t)$, то $(\nu(t), \dot{\nu}(t))$ - траектория в $L_{k c}$. Поэтому можно ограничиться изучением ограничения геодезического потока на компактное многообразие уровня $S M^{n}=\{H=1\}$. В этом случае энтропией геодезического потока называется энтропия его ограничения на $S M^{n}$, и поток называется интегрируемым, если кроме $H=I_{n}$ существует еще $(n-1)$ первых интегралов $I_{1}, \ldots, I_{n-1}$ таких, что первые интегралы $\left\{I_{j}\right\}$ инволютивны $\left(\left\{I_{j}, I_{k}\right\}=0\right.$ при $\left.1 \leqslant j, k \leqslant n\right)$ в окрестности $S M^{n}$ и функционально независимы почти всюду на $S M^{n}$.

Проблема нахождения топологических препятствий к интегрируемости была поставлена Козловым, который нашел все замкнутые двумерные многообразия, допускающие (вещественно) аналитические римановы метрики, чьи геодезические потоки имеют полный набор аналитических первых интегралов [1]. Для многообразий большей размерности препятствия к аналитической интегрируемости были найдены в [2], [3]. Заметим, что все эти результаты в действительности описывают препятствия к достаточно простому геометрическому поведению системы, которое в [2] было названо геометрической простотой и гарантируется аналитической интегрируемостью.

Патернайн предложил сначала искать препятствия к существованию геодезического потока с нулевой топологической энтропией, а затем искать препятствия к такой интегрируемости, которая гарантировала бы равенство нулю энтропии [4], [5] (см. также [6]).

Недавно Батлер нашел красивый трюк, позволивший ему построить такие геодезические потоки аналитических метрик на ряде нильмногообразий, что эти потоки интегрируемы с помощью $C^{\infty}$-первых интегралов и имеют нулевую топологическую энтропию, но неинтегрируемы с помощью аналитических первых интегралов [7]. Последний факт доказывается использованием препятствий к аналитической интегрируемости, найденных в [2], [3], что показывает, что в общем случае результаты этих работ не обобщаются на $C^{\infty}$-интегрируемость.

Батлер строит метрику на бесконечном произведении $T^{n} \times \mathbb{R}$ тора и прямой, инвариантную относительно действия $\mathbb{Z}$ вида $(x, y) \rightarrow(A x, y+1)$, где $A$ - автоморфизм тора, и опускает ее на компактное фактор-пространство $\left(T^{n} \times \mathbb{R}\right) / \mathbb{Z}$. Полученная метрика и является искомой. Простейшим примером является случай $n=2$, когда автоморфизм тора задается матрицей

$$
\left(\begin{array}{ll}
1 & 1 \\
0 & 1
\end{array}\right)
$$

приводящий к трехмерному нильмногообразию [7].

Но эта конструкция, примененная к другим матрицам $A$, приводит к более интересным примерам. Пусть $n=2$ и

$$
A=\left(\begin{array}{ll}
2 & 1 \\
1 & 1
\end{array}\right)
$$

Работа выполнена при поддержке Российского фонда фундаментальных исследований (гранты № № 96-15-96868, 98-01-00240 (А. В. Б.) и № № 96-15-96877, 98-01-00749 (И. А. Т.)). 
Определим на $T^{2} \times \mathbb{R}$ с координатами $\left(\left(x^{1}, x^{2}\right) \bmod \mathbb{Z}^{2}, x^{3}\right)$ метрику $g_{j k} d x^{j} d x^{k}$ по формулам $g_{33}=1, g_{13}=g_{23}=0$,

$$
\left(\begin{array}{ll}
g_{11} & g_{12} \\
g_{21} & g_{22}
\end{array}\right)=\exp \left(-x^{3} G_{0}^{\top}\right) \exp \left(-x^{3} G_{0}\right), \text { где } \exp G_{0}=A,
$$

и опустим ее на фактор-многообразие $M_{A}=\left(T^{2} \times \mathbb{R}\right) /\left\{\left(X, x^{3}\right) \sim\left(A X, x^{3}+1\right)\right\}$, где $X=$ $\left(x^{1}, x^{2}\right)$.

Теорема. 1) Геодезический поток на вещественно-аналитическом римановом многообразии $M_{A}$ интегрируем с помощью $C^{\infty}{ }_{-}$первьх интегралов

$$
I_{1}=p_{1}^{2}-p_{1} p_{2}-p_{2}^{2}, \quad I_{2}=\exp \left(-\frac{1}{I_{1}^{2}}\right) \sin \left(\frac{\log \left|p_{1}-\frac{1+\sqrt{5}}{2} p_{2}\right|}{\log \lambda}\right), \quad \text { де } \quad \lambda=\frac{3+\sqrt{5}}{2},
$$

и $I_{3}=\frac{1}{2} g^{j k} p_{j} p_{k}$, которые функционально независимы на подмножестве полной меры в $S M_{A}$

2) геодезический поток на $M_{A}$ не интегрируем с помощью аналитических первых интегралов;

3) фундаментальная группа $\pi_{1}\left(M_{A}\right)$ имеет әкспоненциальный рост;

4) сдвиг вдоль траектории потока за единичное время переводит тор $T^{2}=$ $\left\{p_{1}=p_{2}=x^{3}=0\right\} \subset S M_{A}$ в себя посредством аносовского автоморфизма, задаваемого матрищей $A$;

5) геодезический поток имеет положительную топологическую әнтропию и нулевую әнтропию по мере Лиувилля.

Насколько нам известно, это первый пример геодезического потока, для которого выполнено утверждение 5 теоремы, и первый пример интегрируемого потока с положительной топологической энтропией.

Появление аносовского автоморфизма с качественной точки зрения, по-видимому, объясняется следующим свойством метрики: ее секционна кривизна вдоль любой двумерной площадки $\sigma$, порожденной вектором, касательным к слою $x^{3}=$ const, и вектором, ортогональным этому слою, отрицательна, $K_{\sigma}=-\log ^{2} \lambda$, что и приводит к экспоненциальному разбеганию траекторий, ортогональных слоям.

Равенство нулю энтропии по мере Лиувилля следует из того, что множество полной меры заполнено инвариантными торами, и, следовательно, на нем показатели Ляпунова равны нулю.

Заметим, что так как $\pi_{1}\left(M_{A}\right)$ содержит подгруппу, изоморфную $\mathbb{Z} \oplus \mathbb{Z}$, то $M_{A}$ не допускает метрик отрицательной секционной кривизны. Секционные кривизны данной метрики вдоль площадок, касательных к слоям $\left\{x^{3}=\right.$ const $\}$, положительны и всюду равны $\log ^{2} \lambda$, тензор Риччи неотрицательно определен, а скалярная кривизна положительна и равна $2 \log ^{2} \lambda$.

Подробное доказательство будет опубликовано отдельно.

Авторы благодарят Л. Батлера за присылку препринта [7] и И. К. Бабенко и Г. Патернайна за полезные обсуждения.

\section{СПИСОК ЛИТЕРАТУРЫ}

[1] Козлов В. В. // Докл. АН СССР. 1979. Т. 249. №6. С. 1299-1302. [2] Тайманов И. А. // Изв. АН СССР. Сер. матем. 1987. Т. 51. №2. С. 429-435. [3] Тайманов И. А. // Матем. заметки. 1988. T. 44. № 4. C. 283-284. [4] Paternain G. P. // Ergodic Theory Dynam. Systems. 1992. V. 12. № 2. P. 109-122. [5] Paternain G. P. // J. Geom. Phys. 1994. V. 13. № 3. P. 289-298. [6] Тайманов И. А. // Труды МИРАН. 1994. Т. 205. С. 150-163. [7] Butler L. // Math. Preprint \# 1998-8, Queen's University at Kingston, Canada, November, 1998.

Московский государственный университет им. М. В. Ломоносова; Институт математики СО РАН
Принято редколлегией 21.07.1999 